\title{
A novel TNM staging system for gastric cancer based on the metro- ticket paradigm: a comparative study with the AJCC-TNM staging system
}

\author{
Jun Lu' ${ }^{1,2} \cdot$ Zhi-Fang Zheng ${ }^{1,2} \cdot$ Wei Wang $^{3} \cdot$ Jian-Wei Xie ${ }^{1,2} \cdot$ Jia-Bin Wang ${ }^{1,2,3,4} \cdot$ Jian-Xian Lin ${ }^{1,2,3,4} \cdot$ Qi-Yue Chen ${ }^{1,2}$.

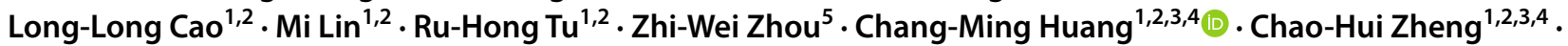 \\ Ping $\mathrm{Li}^{1,2,3,4}$
}

Received: 2 June 2018 / Accepted: 24 November 2018 / Published online: 5 January 2019

(c) The International Gastric Cancer Association and The Japanese Gastric Cancer Association 2019

\begin{abstract}
Background The metro-ticket prognostic tool for hepatocellular carcinoma has been proven to predict outcome, but a similar concept has not been investigated for GC. The objective of the current study was to apply the principles of the metro-ticket paradigm to develop a novel TNM staging system (nTNM) for gastric cancer (GC).

Methods The nTNM considered the distance from the origin on a Cartesian plane incorporating the $\mathrm{pN}$ ( $x$-axis) and pT (y-axis) stages. GC patients undergoing radical resection at Fujian Medical University Union Hospital (FMUUH) $(n=4267)$ were included. The nTNM was validated using 2 external cohorts from the Sun Yat-sen University Cancer Center (SYSUCC) $(n=1800)$ and Surveillance, Epidemiology, and End Results (SEER) $(n=3227)$ databases.

Results nTNM classes with the same distance from the origin have same stage; the stage increases with this distance. Among all patients, $48.0 \%(n=2049)$ were restaged in the nTNM compared with the 7th edition of the AJCC-TNM classification; $26.2 \%(n=1116)$ were downstaged in the nTNM compared with the 8th edition. The nTNM provides significant survival differences between stages (all $P<0.001$ ). The survival difference between stages IB and IIA was especially large for the nTNM $(P<0.001)$ compared to the 7 th and 8 th editions $(P=0.073)$. The concordance index and hazard ratio increased successively with the nTNM stage. Similar findings were observed in both external cohorts.

Conclusion Compared with the AJCC-TNM classification, the nTNM for GC is easier to remember and provides some improvements; therefore, the nTNM may be considered for adoption in future editions of the AJCC-TNM classification.
\end{abstract}

Keywords Gastric cancer $\cdot$ AJCC $\cdot$ TNM staging system $\cdot$ Metro-ticket

Jun Lu, Zhi-Fang Zheng, and Wei Wang have contributed equally to this work, and should be considered co-first authors.

\section{Electronic supplementary material The online version of this} article (https://doi.org/10.1007/s10120-018-00904-w) contains supplementary material, which is available to authorized users.

Zhi-Wei Zhou

zhouzhw@sysucc.org.cn

$\triangle$ Chang-Ming Huang

hcmlr2002@163.com

$\triangle$ Chao-Hui Zheng

wwkzch@163.com

Ping Li

pingli811002@163.com

1 Department of Gastric Surgery, Fujian Medical University Union Hospital, No. 29 Xinquan Road, Fuzhou 350001,

Fujian Province, China
2 Department of General Surgery, Fujian Medical University Union Hospital, Fuzhou, China

3 Key Laboratory of Ministry of Education of Gastrointestinal Cancer, Fujian Medical University, Fuzhou, China

4 Fujian Key Laboratory of Tumor Microbiology, Fujian Medical University, Fuzhou, China

5 Department of Gastric Surgery, Sun Yat-sen University Cancer Center, 651 Dongfeng Road East, Guangzhou 510060, Guangdong, China 


\section{Introduction}

The TNM staging system is the most important prognostic measure for gastric cancer (GC). To predict the prognosis more accurately, the American Joint Committee on Cancer (AJCC) has revised the TNM staging system for GC several times in past decades. The 8th edition (8th) of the AJCC staging system was released in October 2016 and was recommended as a replacement for the older version in 2018 [1]. Research on the prognostic performance of the latest version has been gradually reported [2-6]. In our opinion, the 8th AJCC-TNM staging system can better distinguish the prognosis of GC patients. However, the major change from the former system is the separation of N3a (7-15 positive regional lymph nodes) and N3b ( $>15$ positive regional lymph nodes) in the final staging classification [1], and no changes were made to stages I and II except for T1N3bM0 (changed from IIB in the 7th edition to IIIB in the 8th edition), which may limit the prognostic ability of the newest edition of the AJCC staging system.

The metro-ticket paradigm was first proposed by Mazzaferro [1] in hepatocellular carcinoma (HCC) in 2007 and developed into a new predictive tool termed the metro-ticket system for HCC patients after liver transplantation [8]. The new prognostic tool has been proven to accurately stratify HCC patients for long-term survival, with the prognosis worsening as tumor size and number increase, just as longer trips on the "Metro" result in a higher "ticket" price. The HCC new prognostic tool combines tumor size with number, representing a paradigm shift from a dichotomous to a continuous prognostic stratification of patients with HCC [7, 8]. Based on the principles of the metro-ticket paradigm, we assume that the application of the metro-ticket paradigm to the TNM staging system may improve the prognostic performance of the TNM staging system.

Therefore, using international, multicenter, large sample data, this study aims (1) to apply the metro-ticket paradigm to TNM classification for the first time and to develop a novel TNM staging system (nTNM) for GC; (2) to compare the prognostic performance of the nTNM with the AJCC-TNM staging system (7th and 8th editions); (3) to confirm the validity of the nTNM using two external validation cohorts from China and the United States (US).

\section{Materials and methods}

\section{Patient population}

Patients who underwent radical resection for primary GC at Fujian Medical University Union Hospital (FMUUH) between January 1995 and October 2014 were identified from a prospective database. Patients excluded were (1) those who underwent preoperative chemotherapy or radiotherapy; (2) those with a number of examined lymph nodes (eLNs) $\leq 15$; (3) those with postoperative death within 3 months (based on the landmark analysis [9] to minimize immortal time bias); (4) patients with cancer of the esophagogastric junction (EGJ), as it was not recommended for use of the stomach schema in the 8th AJCC-TNM staging system; (5) patients with the other synchronous malignancies. Finally, 4267 patients were included in this study (Supplementary Fig. 1). The type of surgical resection and the extent of lymph-node dissection were selected according to the Japanese Gastric Cancer Association [10, 11]. According to the patient's wishes and physical condition, fluoride-based adjuvant chemotherapy was recommended for the most patients with stage II or III GC in our center. The $\mathrm{T}$ stage, $\mathrm{N}$ stage, and final stage of the all study patients were classified according to both the 7th and 8th AJCC-TNM classification. The median follow-up time was 71.0 months. The study was approved by the FMUUH Institutional Review Board.

\section{Proposal of the nTNM based on the metro-ticket paradigm}

Based on a concept similar to the metro-ticket paradigm for $\mathrm{HCC}$, we proposed the nTNM by combining the pT stage and $\mathrm{pN}$ stage in which we maintained the $\mathrm{pT}$ and $\mathrm{pN}$ definitions. Therefore, the nTNM was defined as the distance from the origin on a Cartesian plane that incorporated 2 variables: pN stage ( $x$-axis) and pT stage ( $y$-axis). The Pythagorean theorem was then used to calculate the distance of any given point from the origin of the plane $(0,0)$, whereby $\left[(\mathrm{nTNM})^{2}=(\mathrm{pN})^{2}+(\mathrm{pT})^{2}\right]$ (Fig. 1a). The distribution of the nTNM is similar to that of the AJCC-TNM staging system from IA to IIIC (Fig. 1a). The prognostic performance of the nTNM was compared with that of the AJCC-TNM staging system (7th edition and 8th edition).

\section{External validation population}

Two additional external validation data sets that satisfied the aforementioned inclusion and exclusion criteria were obtained from the Sun Yat-sen University Cancer Center (SYSUCC) database from January 1994 to December 2012 and from the Surveillance, Epidemiology, and End Results (SEER) database from January 1988 to December 2008 (Supplementary Fig. 2). The Institutional Review Boards of SYSUCC approved the study. The median follow-up time of the patients in the SYSUCC database was 58.0 months. 

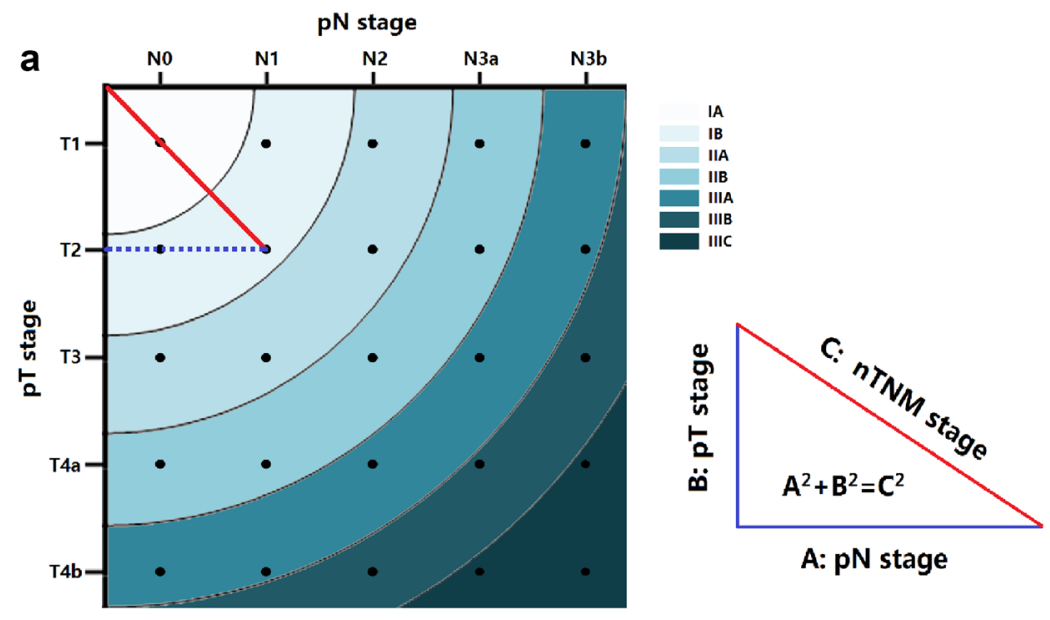

A: pN stage b

\begin{tabular}{|c|c|c|c|c|c|}
\hline $\begin{array}{c}\text { nTHI } \\
\text { stage }\end{array}$ & H0 & H1 & H2 & H3a & H3b \\
\hline T1 & IA & IB & IIA & IIB & IIIA \\
\hline T2 & IB & IB & II\& & IIB & IIIA \\
\hline T3 & IIA & IIA & IIB & IIIA & IIIB \\
\hline T4a & IIB & IIB & IIIA & IIIB & IIIC \\
\hline T4b & III & III & IIIB & IIIC & IIIC \\
\hline
\end{tabular}

d

\begin{tabular}{|c|c|c|c|c|c|}
\hline $\begin{array}{c}\text { 8thTHI } \\
\text { stage }\end{array}$ & H0 & H1 & H2 & H3a & H3b \\
\hline T1 & IA & IB & IIA & IIB & IIIB \\
\hline T2 & IB & IIA & IIB & IIIA & IIIB \\
\hline T3 & IIA & IIB & IIIA & IIIB & IIIC \\
\hline T4a & IIB & IIIA & IIIA & IIIB & IIIC \\
\hline T4b & IIIA & IIIB & IIIB & IIIC & IIIC \\
\hline
\end{tabular}

Fig. 1 a Novel TNM staging system (nTNM) was defined using the distance from the origin on a Cartesian plane that incorporated 2 variables: $\mathrm{pN}$ stage ( $x$-axis) and pT stage ( $y$-axis). The Pythagorean theorem was then used to calculate the distance of any given point from

\section{Statistical analysis}

Overall survival (OS) was calculated from the date of surgery until the date of death or last follow-up. OS was estimated using the Kaplan-Meier method and the log-rank test was used to assess differences in OS. The concordance index (C-index) was calculated to evaluate the discriminatory powers of the competing staging systems. Cox proportional hazard modeling was used to assess the relative impacts of the disease stages on survival. Survival estimates are reported as hazard ratios (HRs) with $95 \%$ confidence intervals (95\% CIs). Variables with a $P<0.05$ in the univariable analysis were included in the multivariable analysis. All analyses were two-sided and values of $P<0.05$ were considered to indicate statistical significance. The statistical analyses were performed using SPSS version 22.0 (IBM Corporation, Chicago, IL) and R version 3.4.0 (Bell Laboratories, Murray Hill, NJ). the origin of the plane $(0,0)$, whereby $\left[(\mathrm{nTNM})^{2}=(\mathrm{pN})^{2}+(\mathrm{pT})^{2}\right] . \mathbf{b}$ nTNM classification. $\mathbf{c} 7$ th edition of the AJCC-TNM classification. $\mathbf{d}$ 8th edition of the AJCC-TNM classification

\section{Results}

\section{Patient characteristics}

A total of 4267 patients from FMUUH were included in this study. The baseline characteristics are presented in Table 1 . The mean age at diagnosis was 59.5 years, and the majority of the patients $(75.8 \%)$ were male. Fifty-nine percent of the patients had a tumor larger than $50 \mathrm{~mm}$. Approximately half of the patients had tumors located in lower sites of the stomach, and more than half of the patients were diagnosed with stage III GC (by the 7th or 8th AJCC-TNM classification). In addition, 2730 patients (55.6\%) underwent total gastrectomy. The pathological type in 2317 patients $(54.3 \%)$ was poorly differentiated or undifferentiated. The mean number of eLNs was 32 and the mean number of positive lymph nodes was 7.2. Moreover, 2459 patients (57.6\%) received postoperative adjuvant chemotherapy. 
Table 1 Patient and tumor characteristics

\begin{tabular}{|c|c|c|c|c|c|c|}
\hline \multirow[t]{2}{*}{ Characteristic } & \multicolumn{2}{|c|}{$\begin{array}{l}\text { FMUUH training set } \\
(n=4267)\end{array}$} & \multicolumn{2}{|c|}{$\begin{array}{l}\text { SYSUCC validation } \\
\text { set }(n=1800)\end{array}$} & \multicolumn{2}{|c|}{$\begin{array}{l}\text { SEER validation set } \\
(n=3227)\end{array}$} \\
\hline & Mean or $n$ & SD or $\%$ & Mean or $n$ & $\mathrm{SD}$ or $\%$ & Mean or $n$ & $\mathrm{SD}$ or $\%$ \\
\hline Age, mean (SD), year & 59.5 & 11.4 & 55.9 & 12.0 & 66.7 & 13.4 \\
\hline \multicolumn{7}{|l|}{ Gender } \\
\hline Male & 3235 & 75.8 & 1202 & 66.8 & 1725 & 53.5 \\
\hline Female & 1032 & 24.2 & 598 & 33.2 & 1502 & 46.5 \\
\hline \multicolumn{7}{|l|}{ Tumor size, $\mathrm{mm}$} \\
\hline$\leq 50$ & 2519 & 59.0 & 1231 & 68.4 & 1316 & 40.8 \\
\hline$>50$ & 1748 & 41.0 & 569 & 31.6 & 1606 & 49.8 \\
\hline Unknown & l & / & / & l & 305 & 9.4 \\
\hline \multicolumn{7}{|l|}{ Tumor site } \\
\hline Cardia/fundus & 1183 & 27.7 & 523 & 29.1 & 152 & 4.7 \\
\hline Body & 788 & 18.5 & 362 & 20.1 & 395 & 12.2 \\
\hline Antrum/pylorus & 1709 & 40.1 & 830 & 46.1 & 1137 & 35.2 \\
\hline Overlapping regions & 587 & 13.7 & 85 & 4.7 & 388 & 12.1 \\
\hline Lesser/greater curvature & l & l & l & l & 816 & 25.3 \\
\hline Stomach NOS & I & I & I & I & 339 & 10.5 \\
\hline \multicolumn{7}{|l|}{ pT stage } \\
\hline $\mathrm{T} 1$ & 761 & 17.8 & 217 & 12.1 & 644 & 20.0 \\
\hline $\mathrm{T} 2$ & 454 & 10.6 & 222 & 12.3 & 380 & 11.8 \\
\hline $\mathrm{T} 3$ & 845 & 19.8 & 345 & 19.2 & 898 & 27.8 \\
\hline $\mathrm{T} 4 \mathrm{a}$ & 1682 & 39.5 & 881 & 48.9 & 1030 & 31.9 \\
\hline $\mathrm{T} 4 \mathrm{~b}$ & 525 & 12.3 & 135 & 7.5 & 275 & 8.5 \\
\hline \multicolumn{7}{|l|}{ pN stage } \\
\hline No & 1287 & 30.2 & 571 & 31.7 & 1175 & 36.4 \\
\hline N1 & 597 & 14.0 & 271 & 15.1 & 395 & 12.2 \\
\hline $\mathrm{N} 2$ & 741 & 17.4 & 310 & 17.2 & 464 & 14.4 \\
\hline $\mathrm{N} 3 \mathrm{a}$ & 971 & 22.7 & 401 & 22.3 & 658 & 20.4 \\
\hline $\mathrm{N} 3 \mathrm{~b}$ & 671 & 15.7 & 247 & 13.7 & 535 & 16.6 \\
\hline \multicolumn{7}{|l|}{ AJCC 7th pTNM stage } \\
\hline IA & 618 & 14.5 & 167 & 9.3 & 531 & 16.4 \\
\hline IB & 313 & 7.3 & 144 & 8.0 & 261 & 8.1 \\
\hline IIA & 345 & 8.1 & 153 & 8.5 & 362 & 11.2 \\
\hline IIB & 459 & 10.8 & 291 & 16.2 & 354 & 11.0 \\
\hline IIIA & 396 & 9.3 & 209 & 11.6 & 303 & 9.4 \\
\hline IIIB & 739 & 17.3 & 308 & 17.1 & 619 & 19.2 \\
\hline IIIC & 1397 & 32.7 & 528 & 29.3 & 797 & 24.7 \\
\hline \multicolumn{7}{|l|}{ AJCC 8th pTNM stage } \\
\hline IA & 618 & 14.5 & 167 & 9.3 & 531 & 16.4 \\
\hline IB & 313 & 7.3 & 144 & 8.0 & 261 & 8.1 \\
\hline IIA & 345 & 8.1 & 153 & 8.5 & 362 & 11.2 \\
\hline IIB & 455 & 10.7 & 291 & 16.2 & 346 & 10.7 \\
\hline IIIA & 791 & 18.5 & 384 & 21.3 & 518 & 16.1 \\
\hline IIIB & 920 & 21.6 & 380 & 21.1 & 603 & 18.7 \\
\hline IIIC & 825 & 19.3 & 281 & 15.6 & 606 & 18.8 \\
\hline \multicolumn{7}{|l|}{ Type of surgery } \\
\hline Total gastrectomy & 2370 & 55.6 & 375 & 20.8 & 2336 & 73.4 \\
\hline Non-total gastrectomy & 1897 & 44.4 & 1425 & 79.2 & 891 & 27.6 \\
\hline \multicolumn{7}{|l|}{ Histology } \\
\hline G1/G2 & 1816 & 42.6 & 326 & 18.2 & 858 & 26.6 \\
\hline G3/G4 & 2317 & 54.3 & 1474 & 81.8 & 2201 & 68.2 \\
\hline
\end{tabular}


Table 1 (continued)

\begin{tabular}{|c|c|c|c|c|c|c|}
\hline \multirow[t]{2}{*}{ Characteristic } & \multicolumn{2}{|c|}{$\begin{array}{l}\text { FMUUH training set } \\
(n=4267)\end{array}$} & \multicolumn{2}{|c|}{$\begin{array}{l}\text { SYSUCC validation } \\
\text { set }(n=1800)\end{array}$} & \multicolumn{2}{|c|}{$\begin{array}{l}\text { SEER validation set } \\
(n=3227)\end{array}$} \\
\hline & Mean or $n$ & $\mathrm{SD}$ or $\%$ & Mean or $n$ & $\mathrm{SD}$ or $\%$ & Mean or $n$ & $\mathrm{SD}$ or $\%$ \\
\hline Unknown & 134 & 3.1 & / & l & 168 & 5.2 \\
\hline Lymph nodes examined, mean (SD) & 32.0 & 12.0 & 27.7 & 10.1 & 25.9 & 11.0 \\
\hline Positive lymph nodes, mean (SD) & 7.2 & 9.3 & 6.6 & 8.7 & 6.8 & 9.1 \\
\hline \multicolumn{7}{|l|}{ Adjuvant chemotherapy } \\
\hline No & 1808 & 42.4 & Missing & I & Missing & l \\
\hline Yes & 2459 & 57.6 & & I & & I \\
\hline
\end{tabular}

In addition, 1800 patients from the SYSUCC database and 3227 patients from the SEER database were included in this study. In the SYSUCC cohort, the mean age was 55.9 years, and the ratio of male-to-female patients was approximately $2: 1$. The tumor location and pathological stage were similar to those of the FMUUH cohort, but most of them (79.2\%) underwent a subtotal gastrectomy. In the SEER cohort, the mean age was 66.7 years, and the ratio of maleto-female patients was approximately $1: 1$. More than half of the patients were in stage III; most of whom $(73.4 \%)$ were treated with total gastrectomy. Detailed clinicopathological features are shown in Table 1.

\section{The novel TNM staging system (nTNM)}

The nTNM (Fig. 1a, b) is intuitively compared with the AJCC-TNM staging system (Fig. 1c, d). Compared with the 7th AJCC-TNM classification (Fig. 1c), the nTNM stage changed except for stage IA. Upstaging was observed in 2045 patients and downstaging was observed in 4 patients. A total of $48.0 \%(n=2049)$ of the patients were restaged in the nTNM. The distribution of patients in the 7th AJCCTNM classification and the nTNM is shown in Supplementary Table 1.

Compared with the stage from the 8th AJCC-TNM classification (Fig. 1d), the nTNM stage also changed except for stage IA. Downstaging was observed in T1N3bM0, $\mathrm{T} 2-3 \mathrm{~N} 1 \mathrm{bM} 0$ and $\mathrm{T} 4 \mathrm{a}-4 \mathrm{bN} 1 \mathrm{M} 0$ classifications. A total of $26.2 \%$ ( $n=1116$ ) of the patients were restaged in the nTNM. The distribution of patients in the 8th AJCC-TNM classification and the nTNM is shown in Supplementary Table 2.

\section{Comparison of prognostic performance between the nTNM and AJCC-TNM classification}

For the 7th AJCC-TNM classification, the 5-year survival rates for each stage were as follows: IA 94.8\%, IB 89.0\%, IIA $84.6 \%$, IIB $75.9 \%$, IIIA $65.0 \%$, IIIB $52.1 \%$, and IIIC $31.4 \%$ (IB vs IIA: $P=0.073, P<0.05$ for the other stages; Fig. 2a). For the 8th AJCC-TNM classification, the 5-year survival rates for each stage were as follows: IA 94.8\%, IB
$89.0 \%$, IIA $84.6 \%$, IIB $76.1 \%$, IIIA $60.3 \%$, IIIB $40.9 \%$, and IIIC $27.5 \%$ (IB vs IIA: $P=0.073, P<0.05$ for the other stages; Fig. 2b). Meanwhile, for the nTNM, the 5-year survival rates for each stage were as follows: IA $94.8 \%$, IB $88.0 \%$, IIA $80.1 \%$, IIB $67.9 \%$, IIIA $54.4 \%$, IIIB $35.9 \%$, and IIIC $27.4 \%$ (all $P<0.001$; Fig. 2c). Furthermore, the prognostic ability of the nTNM (C-index: $0.744,95 \% \mathrm{CI}$ : $0.729-0.759)$ is superior to that of the 7th (C-index: 0.731 , 95\% CI: 0.717-0.745) and 8th (C-index: $0.741,95 \% \mathrm{CI}$ : 0.727-0.756) AJCC-TNM classification (Fig. 2d).

The univariate Cox analysis showed that the HRs of each stage of the nTNM classification were as follows: IB 2.237, IIA 3.829, IIB 6.801, IIIA 10.008, IIIB 17.402, and IIIC 23.422 (stage IA as the reference). An increase in HR (except for stage IIIC for the AJCC-TNM classification, 8th edition: 23.705 vs nTNM: 23.422) was observed in the nTNM compared with the 7th and 8th AJCC-TNM classification (Supplementary Table 3).

The multivariate Cox analysis showed that the HRs of each stage of the nTNM classification were as follows: IB 2.281, IIA 3.603, IIB 6.464, IIIA 9.186, IIIB 15.871, and IIIC 20.598 (stage IA as the reference). The HRs of the nTNM stages were increased (except for stage IIIC for the AJCC-TNM classification, 8th edition: 20.745 vs nTNM: 20.598) compared with the 7th and 8th AJCC-TNM classification (Table 2).

\section{External validation}

The nTNM was then validated using data obtained from the SYSUCC database and SEER database. The 5-year survival rates for each stage of the nTNM classification in the SYSUCC cohort were as follows: IA $96.1 \%$, IB $90.7 \%$, IIA $82.2 \%$, IIB $76.9 \%$, IIIA $53.8 \%$, IIIB $40 \%$, and IIIC $20.3 \%$ (IIA vs IIB: $P=0.059, P \leq 0.05$ for the other stages; Fig. 3c). The 5-year survival rates for each stage of the nTNM classification in the SEER cohort were as follows: IA $85.4 \%$, IB $75.6 \%$, IIA $59.2 \%$, IIB $36.2 \%$, IIIA $26.4 \%$, IIIB $12.1 \%$, and IIIC $6.2 \%$ (all $P<0.001$; Fig. 4 c). Compared with the 7th (Figs. 3a, 4a) and 8th (Figs. 3b, 4b) AJCC-TNM classification, the nTNM classification 

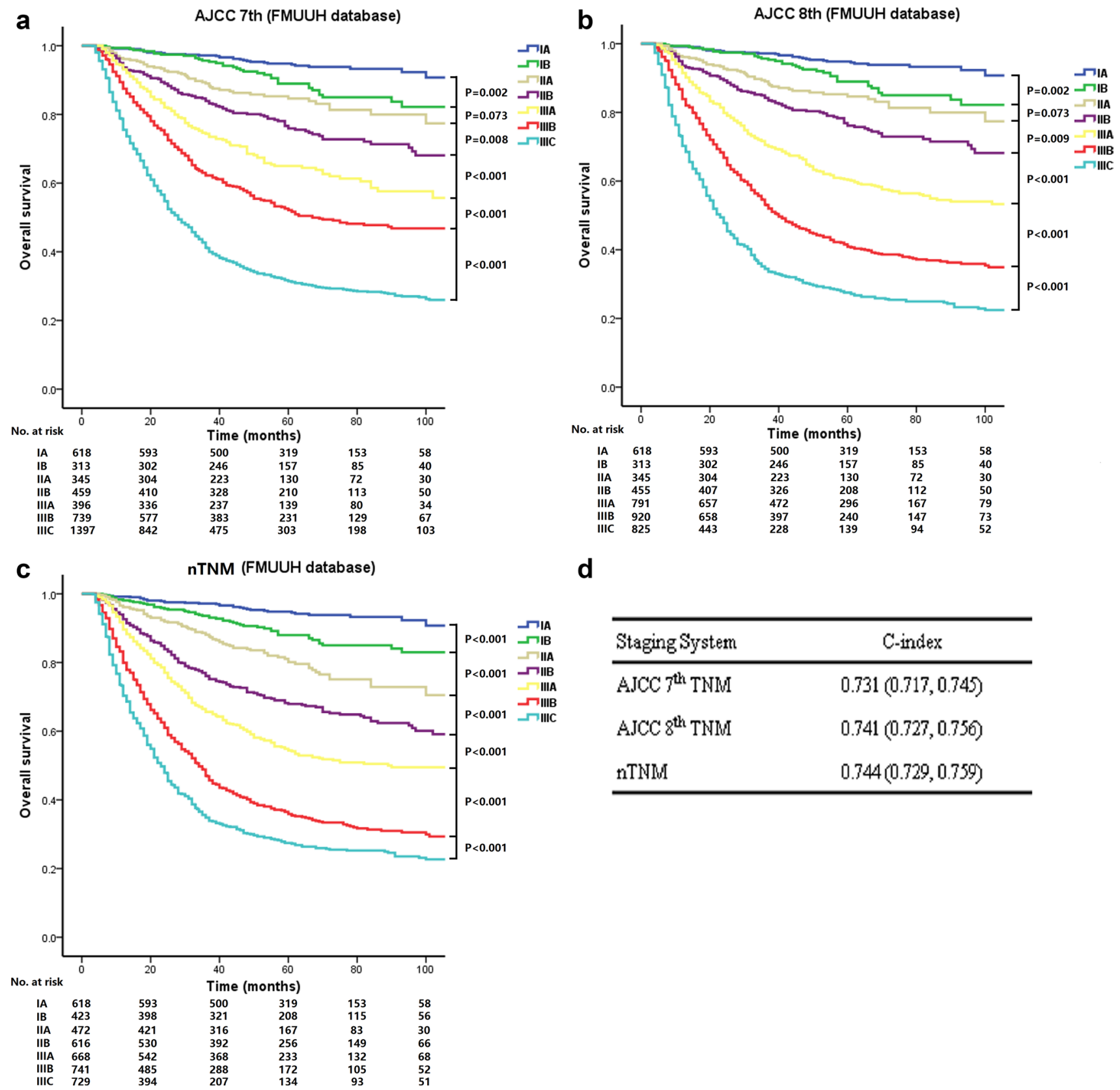

d

\begin{tabular}{lc}
\hline Staging System & C-index \\
\hline AJCC $7^{\text {th }}$ TNM & $0.731(0.717,0.745)$ \\
AJCC $8^{\text {th }}$ TNM & $0.741(0.727,0.756)$ \\
nTNM & $0.744(0.729,0.759)$ \\
\hline
\end{tabular}

Fig. 2 a Kaplan-Meier estimates of overall survival (OS) stratified by the TNM stage using the 7th edition of the AJCC-TNM classification. b Kaplan-Meier estimates of OS stratified by the 8th edition of the

AJCC-TNM classification. c Kaplan-Meier estimates of OS stratified by the nTNM. d C-indexes of different staging systems for GC in the FMUUH training set

\section{Discussion}

showed improved discrimination of the survival curves for both the SYSUCC and SEER cohorts (Figs. 3c, 4c). In addition, the increases in the $\mathrm{C}$-index and HR in the nTNM classification were demonstrated in both external validation cohorts (Figs. 3d, 4d and Supplementary Table 4).

The AJCC-TNM staging system is considered to be the most comprehensive prognostic classification tool for cancer patients. The 8th edition of the AJCC-TNM staging 
Table 2 Results of the multivariate analysis for prognostic factors according to the respective TNM staging systems using the FMUUH database

\begin{tabular}{|c|c|c|}
\hline Variables & Hazard ratio $(95 \% \mathrm{CI})$ & $P$ value \\
\hline \multicolumn{3}{|c|}{ AJCC 7th TNM stage } \\
\hline IA & Reference & $<0.001$ \\
\hline IB & $2.131(1.332-3.410)$ & 0.003 \\
\hline IIA & $2.911(1.880-4.509)$ & $<0.001$ \\
\hline IIB & $4.630(3.137-6.836)$ & $<0.001$ \\
\hline IIIA & $7.000(4.774-10.263)$ & $<0.001$ \\
\hline IIIB & $9.958(6.933-14.302)$ & $<0.001$ \\
\hline IIIC & $17.497(12.244-25.003)$ & $<0.001$ \\
\hline \multicolumn{3}{|c|}{ AJCC 8th TNM stage } \\
\hline IA & Reference & $<0.001$ \\
\hline IB & $2.121(1.325-3.393)$ & 0.002 \\
\hline IIA & $2.906(1.877-4.501)$ & $<0.001$ \\
\hline IIB & $4.556(3.083-6.730)$ & $<0.001$ \\
\hline IIIA & $7.951(5.533-11.425)$ & $<0.001$ \\
\hline IIIB & $13.368(9.341-19.131)$ & $<0.001$ \\
\hline IIIC & $20.745(14.433-29.817)$ & $<0.001$ \\
\hline \multicolumn{3}{|c|}{ nTNM stage } \\
\hline IA & Reference & $<0.001$ \\
\hline IB & $2.281(1.475-3.526)$ & $<0.001$ \\
\hline IIA & $3.603(2.414-5.378)$ & $<0.001$ \\
\hline IIB & $6.464(4.463-9.363)$ & $<0.001$ \\
\hline IIIA & $9.186(6.381-13.224)$ & $<0.001$ \\
\hline IIIB & $15.871(11.063-22.767)$ & $<0.001$ \\
\hline IIIC & $20.598(14.307-29.654)$ & $<0.001$ \\
\hline
\end{tabular}

system for GC was published in October 2016 and is expected to be used in 2018 [1]. The major change is the separation of $\mathrm{N} 3 \mathrm{a}$ and $\mathrm{N} 3 \mathrm{~b}$ in the final staging system [1].

Changes to the latest classification of GC were based on the International Gastric Cancer Association (IGCA) data analysis (84.8\% from Japan and South Korea) [1], and its application to Chinese and American GC populations has been verified by relevant studies [2-4]. Ji et al.'s study showed that the 8th edition is superior to the 7 th edition in terms of homogeneity, discriminatory ability, and monotonicity of gradients for Chinese GC patients [4]. An analysis of 12,041 GC patients from the National Cancer Database (NCDB) demonstrated that although the 8th AJCC-TNM staging system has similar predictive power to the 7th edition (7th edition C-index: 0.720 vs 8 th edition C-index: 0.719 ), the 8th edition can clearly separate data with preservation of group order in US populations [2]. Our previous study found that the 8 th edition provides a better prognosis than the 7th edition in noncardia GC patients with eLNs $>15$ from the SEER and FMUUH databases, but no improvement was found in patients with eLNs $\leq 15$ [3]. In our opinion, the 8th edition is, indeed, better than the 7 th edition in differentiating the prognosis of GC patients, especially those with eLNs $>15$. However, there are still some defects in the 8 th edition $[2,3]$, which suggests that the latest version of the staging system still needs to be improved.

To improve the accuracy of TNM classification for GC, numerous scholars have modified AJCC-TNM staging in different forms [12-14]. Warneke et al. found that the 7th edition is associated with a stage migration in $60 \%$ of patients with esophagogastric cancer and GC. This change did not improve the assessment of patient prognosis, and therefore, a revised "Kiel Proposal of Stage Grouping" was proposed [12]. Marrelli et al. suggested increasing the prognostic weight of the $\mathrm{N}$ status and separating the $\mathrm{N} 3 \mathrm{a}$ and $\mathrm{N} 3 \mathrm{~b}$ categories for stage grouping [13]. Jung et al. combined the 7th edition $\mathrm{pT}$ classification and 6th edition $\mathrm{pN}$ classification to improve the prognostic ability [14]. However, the previous studies were carried out by different combinations of $\mathrm{pT}$ and $\mathrm{pN}$ classifications, which did not change the nature of the TNM classification, and most of the modified TNM classifications have not been verified by external data. In the current study, the principle of the metro-ticket paradigm $[7,8]$ was applied to TNM staging for the first time, and a novel design concept of TNM staging for GC was established.

The metro-ticket system was first used for HCC [7, 8]. Later, the principle was applied to patients undergoing hepatic resection of colorectal liver metastasis (CRLM), thus establishing a new tumor burden score model that can accurately distinguish the prognosis of CRLM patients [15]. Similar to the models for HCC and CRLM, we modeled the pT stage and pN stage on a Cartesian plane and forecasted the OS of patients undergoing radical resection of GC based on this model (Fig. 1a). Figuratively, each point on the chart may be said to represent a potential "destination", while the origin of the Cartesian plane $(0,0)$ is considered as a central "Metro" station. The longer the trip away from the origin (increased $\mathrm{pT}$ and $\mathrm{pN}$ stage), the higher the price of the "ticket" (reduced survival) $[8,15]$.

Compared with the stages of the 7th and 8th AJCC-TNM classification, the stages of the nTNM classification changed except for stage IA. Although the nTNM in this study did not have a significantly increased $\mathrm{C}$-index, a finding similar to results from the previous studies $[2,16]$, the nTNM is significantly superior to the AJCC-TNM staging system in some respects. First, the nTNM provides significant survival differences between each pair of stages (all $P<0.001$ ). In particular, the survival curves of stages IB and IIA in the 7th and 8th AJCC-TNM classification were not well separated, but the separation is improved in the nTNM. This may be important, because different treatment strategies (e.g., adjuvant chemotherapy) should be considered for these patients [17-19]. Second, an increase in HRs was observed in the nTNM stages, which further improves the prognostic performance. Third, nTNM classes with the same distance 

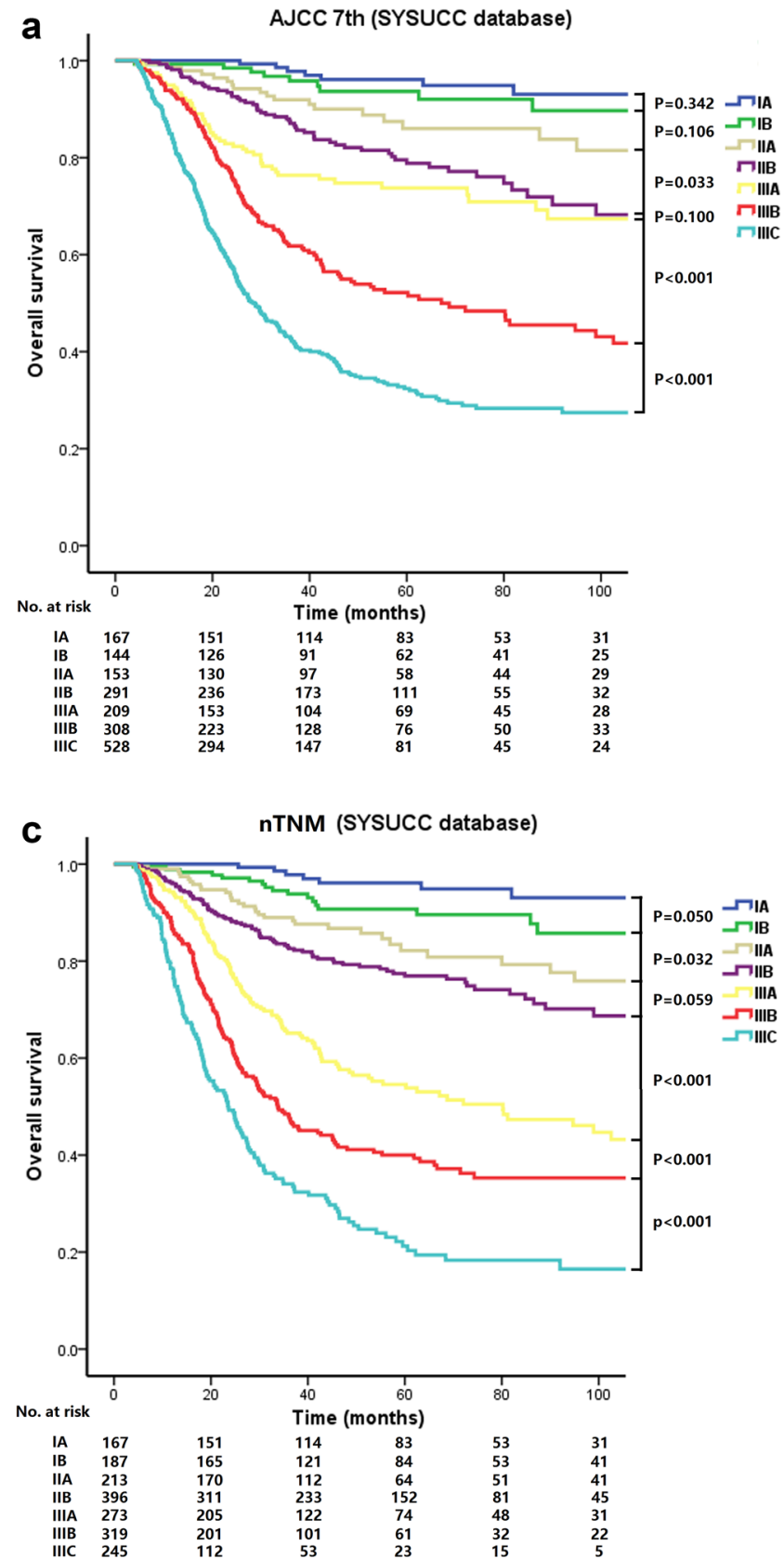

Fig. 3 a Kaplan-Meier estimates of OS stratified with the 7th edition of the AJCC-TNM classification in the SYSUCC external validation cohort. b Kaplan-Meier estimates of OS stratified by the 8th edition of the AJCC-TNM classification in the SYSUCC external validation

from the origin have the same stage, and the stage increases with increasing distance from the origin. For example, both $\mathrm{T} 3 \mathrm{~N} 1 \mathrm{M} 0$ and $\mathrm{T} 2 \mathrm{~N} 2 \mathrm{M} 0$ are classified as stage IIA, and both $\mathrm{T} 4 \mathrm{aN} 2 \mathrm{M} 0$ and $\mathrm{T} 3 \mathrm{~N} 3 \mathrm{aM} 0$ are stage IIIA; this feature makes the nTNM easy to master.

The large sample size and the long follow-up duration of this study provide reliability to the results. Nevertheless, there are several limitations of this study. First, the current

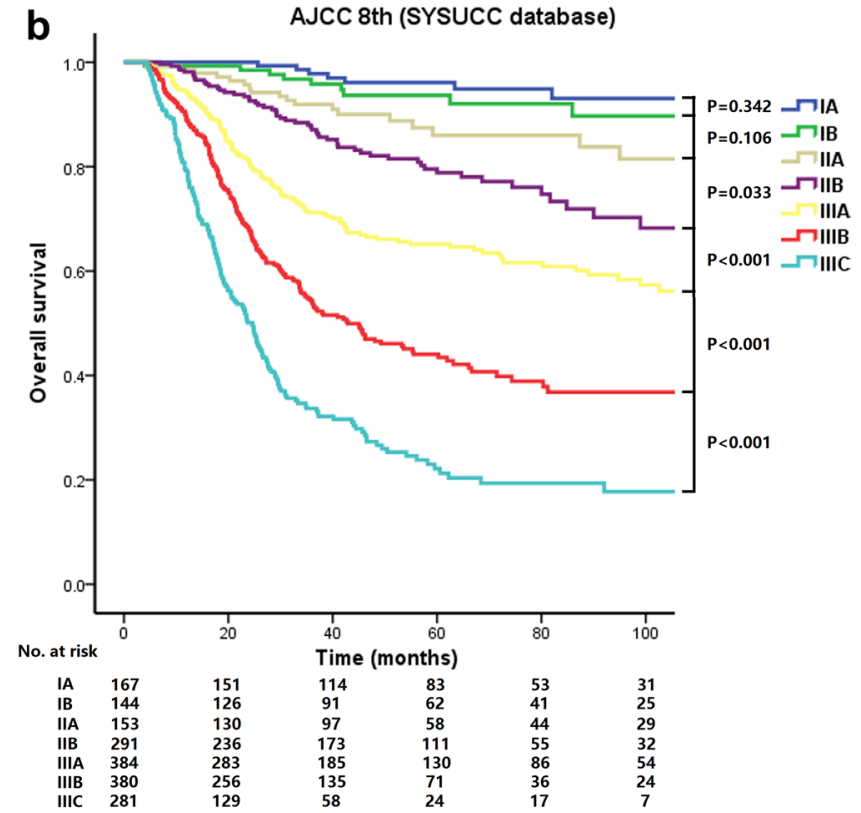

d

\begin{tabular}{lc}
\hline Staging System & C-index \\
\hline AJCC $7^{\text {th }}$ TNM & $0.743(0.718,0.768)$ \\
AJCC $8^{\text {th }}$ TNM & $0.749(0.724,0.774)$ \\
nTNM & $0.751(0.726,0.776)$ \\
\hline
\end{tabular}

cohort. c Kaplan-Meier estimates of OS stratified by the nTNM in the SYSUCC external validation cohort. d C-indexes of different staging systems for GC in the SYSUCC validation set

study is limited by its retrospective nature; prospective studies with large sample sizes are still needed to confirm our results. Second, the SEER database was commonly used for the analysis and verification of staging system as previous studies [3, 16, 20-22], but the proportion of gastric cancer patients with more than 15 examined lymph nodes is not high and there may be inaccurate or missing data in SEER database. Therefore, the results still need to be verified by 

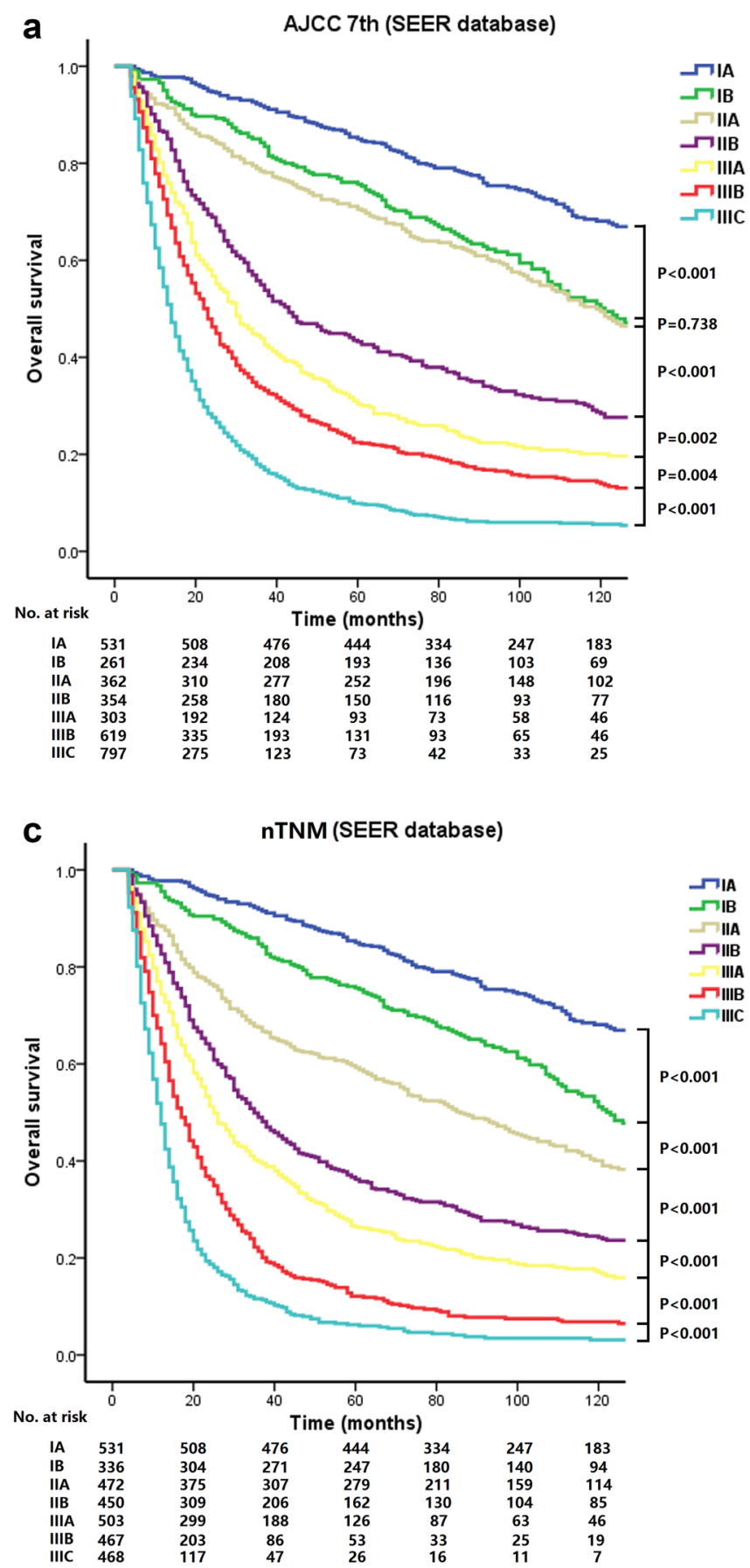

Fig. 4 a Kaplan-Meier estimates of OS stratified with the 7th edition of the AJCC-TNM classification in the SEER external validation cohort. b Kaplan-Meier estimates of OS stratified by the 8th edition of the AJCC-TNM classification in the SEER external validation

another large multicenter data, such as the National Cancer Database. Of course, it would be prudent to confirm our results in a prospective cohort or the second best in another large multiinstitutional database, such as the National Cancer Database. Third, postoperative adjuvant chemotherapy may affect the prognosis of GC patients. The information

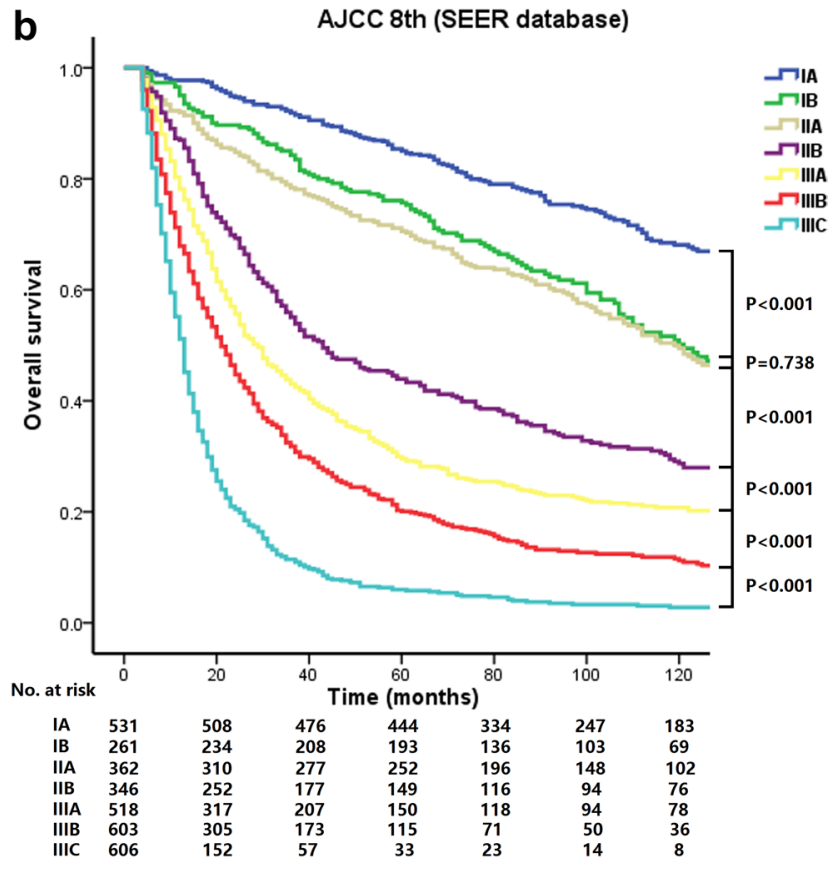

d

\begin{tabular}{lc}
\hline Staging System & C-index \\
\hline AJCC 7 $7^{\text {th }}$ TNM & $0.735(0.721-0.749)$ \\
AJCC 8 $8^{\text {th }}$ THM & $0.742(0.728-0.750)$ \\
nTNM & $0.744(0.730,0.758)$ \\
\hline
\end{tabular}

cohort. c Kaplan-Meier estimates of OS stratified with the nTNM in the SEER external validation cohort. d C-indexes of different staging systems for GC in the SEER validation set

of adjuvant chemotherapy is not available in the external cohorts as previous studies [16, 20,21], which may have a certain impact on the prognostic performance of TNM staging systems. Final, the nTNM may be suitable for the less-optimally treated patients, whether it is suitable for gastric cancer patients in Japan or Korea still needs further 
verification. However, this study applied the metro-ticket paradigm to TNM classification for the first time and developed a novel staging system for GC, which outperformed the AJCC-TNM classification in terms of prognostic performance. Furthermore, utility and validity of the nTNM were verified in two external cohorts.

\section{Conclusions}

In conclusion, the present study demonstrated that the nTNM based on the metro-ticket paradigm was applicable to patients with GC undergoing radical resection. Importantly, the validity of the nTNM was independently confirmed in 2 external cohorts derived from China and the US. The novel TNM staging system is better than the current AJCC-TNM staging system in predicting the long-term survival of GC patients in several aspects and should be considered for potential adoption as a basis of the next version of the AJCC staging system.

Funding This study was funded by the Scientific and Technological Innovation Joint Capital Projects of Fujian Province (2016Y9031), Construction Project of Fujian Province Minimally Invasive Medical Center (No. [2017]171), the second batch of special support funds for Fujian Province Innovation and Entrepreneurship Talents (2016B013), Youth Scientific Research Subject of Fujian Provincial Health and Family Planning Commission (No. 2015-1-37), QIHANG funds of Fujian Medical University (No.2016QH025), and Joint Health Programs of the Provincial Natural Foundation (2015J01464).

\section{Compliance with ethical standards}

Conflict of interest The authors declare that they have no conflict of interest.

Human rights statement and informed consent All procedures followed were in accordance with the ethical standards of the responsible committee on human experimentation (institutional and national) and with the Helsinki Declaration of 1964 and later versions. Informed consent or substitute for it was obtained from all patients for being included in the study.

\section{References}

1. Amin MB, Edge $S$, Greene F, et al. AJCC cancer staging manual, 8th edn. New York: Springer; 2017.

2. In H, Solsky I, Palis B, Langdon-Embry M, Ajani J, Sano T. Validation of the 8th edition of the AJCC TNM staging system for gastric cancer using the national cancer database. Ann Surg Oncol. 2017;24(12):3683-91.

3. Lu J, Zheng ZF, Xie JW, et al. Is the eighth edition of the AJCC TNM staging system sufficiently reasonable for all patients with noncardia gastric cancer? A 12,549 patient international database study. Ann Surg Oncol. 2018;25(7):2002-11.

4. Ji X, Bu Z, Yan Y, et al. The 8th edition of the American Joint Committee on Cancer tumor-node-metastasis staging system for gastric cancer is superior to the 7th edition: results from a Chinese mono-institutional study of 1663 patients. Gastric Cancer. 2017;24(4):643-52.

5. Lu J, Zheng CH, Cao LL, et al. The effectiveness of the 8th American Joint Committee on Cancer TNM classification in the prognosis evaluation of gastric cancer patients: a comparative study between the 7th and 8th editions. Eur J Surg Oncol. 2017;43(12):2349-56.

6. Fang W, Huang K, Chen M, et al. Comparative study of the 7th and 8th AJCC editions for gastric cancer patients after curative surgery. PLOS One. 2017;12(11):e187626.

7. Mazzaferro V. Results of liver transplantation: With or without milan criteria? Liver Transplant. 2007;13(S2):S44-7.

8. Mazzaferro V, Llovet JM, Miceli R, et al. Predicting survival after liver transplantation in patients with hepatocellular carcinoma beyond the Milan criteria: a retrospective, exploratory analysis. Lancet Oncol. 2009;10(1):35-43.

9. Anderson JR, Cain KC, Gelber RD. Analysis of survival by tumor response. J Clin Oncol. 1983;1(11):710-9.

10. Japanese GCA. Japanese classification of gastric carcinoma-2nd English Edition. Gastric Cancer. 1998;1(1):10-24.

11. Japanese GCA. Japanese classification of gastric carcinoma: 3rd English edition. Gastric Cancer. 2011;14(2):101-12.

12. Warneke VS, Behrens HM, Hartmann JT, et al. Cohort study based on the seventh edition of the TNM classification for gastric cancer: proposal of a new staging system. J Clin Oncol. 2011;29(17):2364-71.

13. Marrelli D, Morgagni P, de Manzoni G, et al. Prognostic value of the 7th AJCC/UICC TNM classification of noncardia gastric cancer. Ann Surg. 2012;255(3):486-91.

14. Jung $\mathrm{H}$, Lee $\mathrm{HH}$, Song KY, Jeon HM, Park CH. Validation of the seventh edition of the American Joint Committee on Cancer TNM staging system for gastric cancer. Cancer Am Cancer Soc. 2011;117(11):2371-8.

15. Sasaki K, Morioka D, Conci S, et al. The tumor burden score. Ann Surg. 2018;267(1):132-41.

16. Shi S, Hua J, Liang C, et al. Proposed modification of the 8 th edition of the AJCC staging system for pancreatic ductal adenocarcinoma. Ann Surg. 2018:1.

17. Waddell T, Verheij M, Allum W, Cunningham D, Cervantes A, Arnold D. Gastric cancer: ESMO-ESSO-ESTRO Clinical Practice Guidelines for diagnosis, treatment and follow-up. Ann Oncol. 2013;24(Suppl 6):i57-i63.

18. Japanese GCA. Japanese gastric cancer treatment guidelines 2010 (ver. 3). Gastric Cancer. 2011;14(2):113-23.

19. Lee KG, Lee HJ, Oh SY, et al. Is there any role of adjuvant chemotherapy for T3N0M0 or T1N2M0 gastric cancer patients in stage II in the 7th TNM but stage I in the 6th TNM System? Ann Surg Oncol. 2016;23(4):1234-43.

20. Wang J, Dang P, Raut CP, et al. Comparison of a lymph node ratio-based staging system with the 7th AJCC system for gastric cancer: analysis of 18,043 patients from the SEER database. Ann Surg. 2012;255(3):478-85.

21. Rocken C, Behrens HM. Validating the prognostic and discriminating value of the TNM-classification for gastric cancer-a critical appraisal. Eur J Cancer. 2015;51(5):577-86.

22. Lin J, Lin J, Li P, et al. Which staging system better predicts 10-year survival for gastric cancer? A study using an international multicenter database. Eur J Surg Oncol. 2018;44(8):1205-11.

Publisher's Note Springer Nature remains neutral with regard to jurisdictional claims in published maps and institutional affiliations. 